\title{
Comportamiento mecánico de la aleación AZ31 reforzada con nanofibras de carbono
}

\author{
P. Pérez*, G. Garcés* y P. Adeva*
}

\begin{abstract}
Resumen
En este trabajo se ha estudiado el efecto de la adición de nanofibras de carbono en las propiedades mecánicas de la aleación AZ31 procesada por una ruta pulvimetalúrgica convencional. Se prepararon tres materiales, AZ31, AZ31$1 \%$ C y AZ31-2 \% C. Tras una mezcla mecánica de las nanofibras con los polvos de AZ31, se precompactaron en frío y se extruyeron a $350^{\circ} \mathrm{C}$. Los tres presentan una microestructura recristalizada con un tamaño de grano similar, fino aunque algo heterogéneo. Los tres materiales presentan una débil textura de fibra con el plano basal paralelo a la dirección de extrusión. Las propiedades mecánicas a tracción únicamente se ven afectadas por la presencia de nanofibras a $100{ }^{\circ} \mathrm{C}$ superando los materiales reforzados en un $30 \%$ a los valores de límite elástico y resistencia de la aleación sin reforzar.
\end{abstract}

\section{Mechanical behavior of AZ31 alloy reinforced with carbon nanofibers}

\begin{abstract}
The aim of this study is to investigate the effect of reinforcing AZ31 with carbon nanofibres. The materials AZ31, AZ31-1 \% C y AZ31-2 \% C were produced by a conventional powder metallurgy route consisting of mechanical mixing of nanofibres and powders of AZ31, cool compactation and extrusion at $350^{\circ} \mathrm{C}$. After extrusion the three materials exhibited a recrystallized microstructure of similar grain size, fine and rather inhomogeneous. Furthermore, they presented a weak fibre texture with basal plane parallel to the extrusion direction. The tensile properties were affected by the nanofibres presence only at $100^{\circ} \mathrm{C}$. At this temperature, yield strength and tensile strength were $30 \%$ higher than in the unreinforced alloy.
\end{abstract}

Keywords

Magnesium; Carbon nanofibres; Composite materials; Mechanical properties.

\section{INTRODUCCIÓN}

Es evidente el interés existente, en la última década, por desarrollar aleaciones ligeras de alta resistencia para mejorar la eficiencia energética en la industria del automóvil y del transporte aéreo, a través de la reducción del peso. Por ello, el diseño de aleaciones de magnesio es una línea de investigación muy actual. Los temas de investigación a los que se les está dedicando más atención están relacionados con la solución a alguno de sus problemas, como son su baja resistencia mecánica a temperaturas intermedias, su resistencia a la fluencia y el comportamiento a la corrosión lo que permitiría su uso más extenso en el sector del transporte.

Se están utilizando diferentes estrategias para mejorar las propiedades mecánicas, tales como el control de la composición, diseñando nuevas aleaciones, el control de la microestructura, mediante procesados termomecánicos o, sencillamente, la introducción de un refuerzo que eleve el módulo elástico del material y la resistencia mecánica del material compuesto.

El empleo del carbono, bien como nanotubo o nanofibra, lo convierte en un buen candidato para reforzar aleaciones ligeras, debido a su elevada resistencia y módulo de Young. Además, su elevada superficie específica permite suponer una alta eficacia en la transferencia de carga y una intercara con la matriz muy resistente, frente al movimiento de dislocaciones. El principal inconveniente es su gran tendencia a formar aglomerados, siendo problemático la dispersión homogénea en la matriz. Esta es la base de diferentes estudios para mejorar esta etapa del

\footnotetext{
* Grupo Manoeq. Departamento de Metalurgia Física, Centro Nacional de Investigaciones Metalúrgicas, CENIM-CSIC, Avda. Gregorio del Amo, 8, 28040 Madrid. e-mail: adeva@cenim.csic.es.
} 
proceso, como el de Y. Shimizu et al. ${ }^{[1]}$ o el trabajo de Qianqian Le et al. ${ }^{[2]}$. Otros investigadores han optado por recubrir las fibras para mejorar su mojabilidad $^{[3]}$. Respecto a su efecto como refuerzo de la matriz de magnesio, aún, no está claro. Diferentes estudios discuten sobre el mecanismo de refuerzo de los nanotubos, siendo tres los mecanismos propuestos: transferencia de carga, Orowan y tensiones térmicas generadas por diferencias en los coeficientes de expansión térmica de la matriz y el refuerzo[4].

Este trabajo es un estudio preliminar que los autores están realizando sobre materiales compuestos de matriz aleación de magnesio reforzados con nanofibras de carbono. El objetivo de este trabajo es analizar la influencia que tiene la presencia de nanofibras de carbono en las propiedades mecánicas de una aleación de magnesio, procesada por una ruta pulvimetalúrgica convencional. Esto último, implica la preparación de una mezcla mecánica de nanofibras y polvo de magnesio sin un tratamiento previo de las nanofibras, lo que induce a pensar que puede generar, evidentemente, una distribución heterogénea en la matriz de magnesio.

Se ha seleccionado, como matriz, polvos de la aleación AZ31 solidificados rápidamente, con el fin de poder conocer la interacción refuerzo-matriz y evitar el solapamiento de otros efectos, tales como la presencia de segundas fases, precipitados, etc.

\section{MÉTODO EXPERIMENTAL}

Polvos de aleación AZ31, solidificados rápidamente mediante atomización por argon usando la técnica de electrodo rotatorio (EIGA), con diámetros de partículas menores de $100 \mu \mathrm{m}$ (Fig.1 a)), se mezclaron con nanofibras de carbono, en un molino planetario de alta energía. Las nanofibras fueron suministradas por el Gupo Antolín y como se puede apreciar en la figura 1 b) sus diámetros varían entre 30 y $80 \mathrm{~nm}$; y su longitud puede alcanzar la micra. Se prepararon dos fracciones en volumen, el 1 y $2 \%$. La mezcla mecánica se realizó en tarros de acero inoxidable de $250 \mathrm{ml}$, empleando bolas, también de acero, de $1 \mathrm{~cm}$ de diámetro y una relación masa bolas: masa de material de 3:1. La mezcla mecánica se llevó a cabo a $180 \mathrm{rpm}$ durante $3 \mathrm{~h}$.

Los polvos se precompactaron isostáticamente en frío y, posteriormente, se extruyeron a $350^{\circ} \mathrm{C}$, con una relación de extrusión 1:22. Con fines comparativos, también se preparó la aleación AZ31 sin reforzar, empleando las mismas condiciones, es decir, precompactación en frío y extrusión a $350^{\circ} \mathrm{C}$.
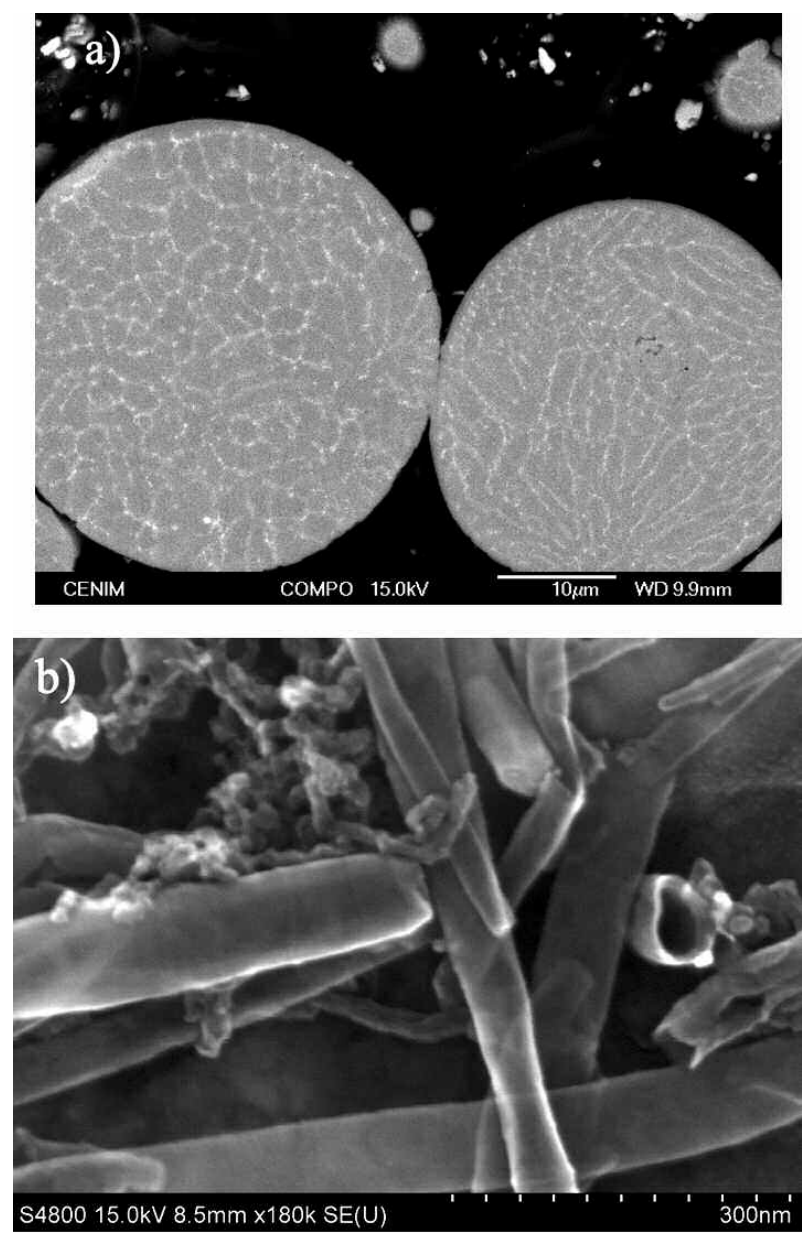

Figura 1. a) Partículas de polvo solidificadas rápidamente de la aleación AZ31. b) Nanofibras de carbono.

\section{Figure 1. a) Rapidly solidified powder particle of AZ31 alloy. b) Carbon nanofibers.}

Se emplearon técnicas de microscopía, tanto óptica como electrónica, para caracterizar microestructuralmente los materiales. También, se empleó difracción de rayos X para determinar la textura de los materiales extruidos. Para ello, se calculó la función de distribución de orientaciones a partir de las

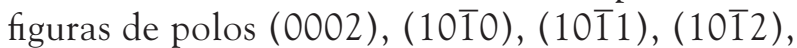
$(10 \overline{1} 3)$ y $(10 \overline{2} 0)$. El sistema de referencia se eligió con el eje $\mathrm{Z}$ paralelo a la dirección de extrusión.

La caracterización mecánica se realizó mediante ensayos de tracción entre temperatura ambiente y $250^{\circ} \mathrm{C}$ empleando una velocidad inicial de deformación de $10^{-4} \mathrm{~s}^{-1}$. Se emplearon probetas cilíndricas de $10 \mathrm{~mm}$ de longitud útil de ensayo y $3 \mathrm{~mm}$ de diámetro, mecanizadas en la dirección de extrusión, realizando tres ensayos para cada condición. Los ensayos se llevaron a cabo en una máquina universal de ensayos continuos y/o dinámicos. 


\section{RESULTADOS Y DISCUSIÓN}

En la figura 2 se presenta la sección longitudinal de las barras extruidas. Se observa, en los tres materiales, una estructura completamente recristalizada con un tamaño de grano fino menor de $5 \mu \mathrm{m}$, con cierta heterogeneidad. El refuerzo, en forma de aglomerados de diferentes tamaños, se observa alineado en la dirección de extrusión y está distribuido, preferentemente, en las fronteras originales de las partículas de polvo. La presencia del refuerzo no parece tener ningún efecto en el tamaño de grano.

Es bien conocida la influencia que tiene, en las propiedades mecánicas de las aleaciones de magnesio, la orientación de sus granos. Así, el magnesio extruido tiene una fuerte anisotropía mecánica que se manifiesta por la inhibición del deslizamiento basal y del maclado durante la tracción en la dirección de extrusión provocando el aumento del límite elástico ${ }^{[5]}$. Por ello, se ha realizado el análisis de la textura de los tres materiales extruidos. Los resultados indican que tanto el material sin reforzar como los reforzados presentan una débil textura de fibra con el plano (0001) paralelo a la dirección de extrusión, como es habitual en el magnesio laminado o extruido. En la figura 3 se presentan las figuras de polos del plano basal (0001) y prismático ( $10 \overline{1} 0$ ) para la aleación AZ31 y para el material reforzado con el 2 \% de nanofibras. Esta figura indica que aunque los dos materiales muestran una textura de fibra débil, la del material monolítico es, ligeramente, más intensa. En la figura 4 se compara la textura de los materiales aquí estudiados con los de la misma aleación procesada por colada y extrusión y se deduce que el procesado pulvimetalúrgico de este material, tanto del monolítico como de los reforzados, disminuye la textura de extrusión, lo cual ha de estar relacionado con el tamaño de grano más fino y homogéneo de los polvos solidificados rápidamente. Se observa que la presencia de las nanofibras no tiene un gran efecto en la textura.

El comportamiento a tracción de los materiales se presenta en la figura 5 e indica que los tres materiales muestran curvas similares, presentando valores muy próximos de límite elástico y resistencia a la tracción, en todo el intervalo de temperaturas estudiado, salvo a $100^{\circ} \mathrm{C}$. A esta temperatura, los materiales reforzados alcanzan valores más elevados de ambas propiedades, siendo ligeramente superior el límite elástico en el caso del material con menor fracción en volumen de refuerzo. Hay que señalar, también, que las aleaciones reforzadas son menos dúctiles en todo el intervalo de temperaturas estudiado.

Los valores de límite elástico, resistencia máxima y alargamiento a rotura de los tres materiales se muestran
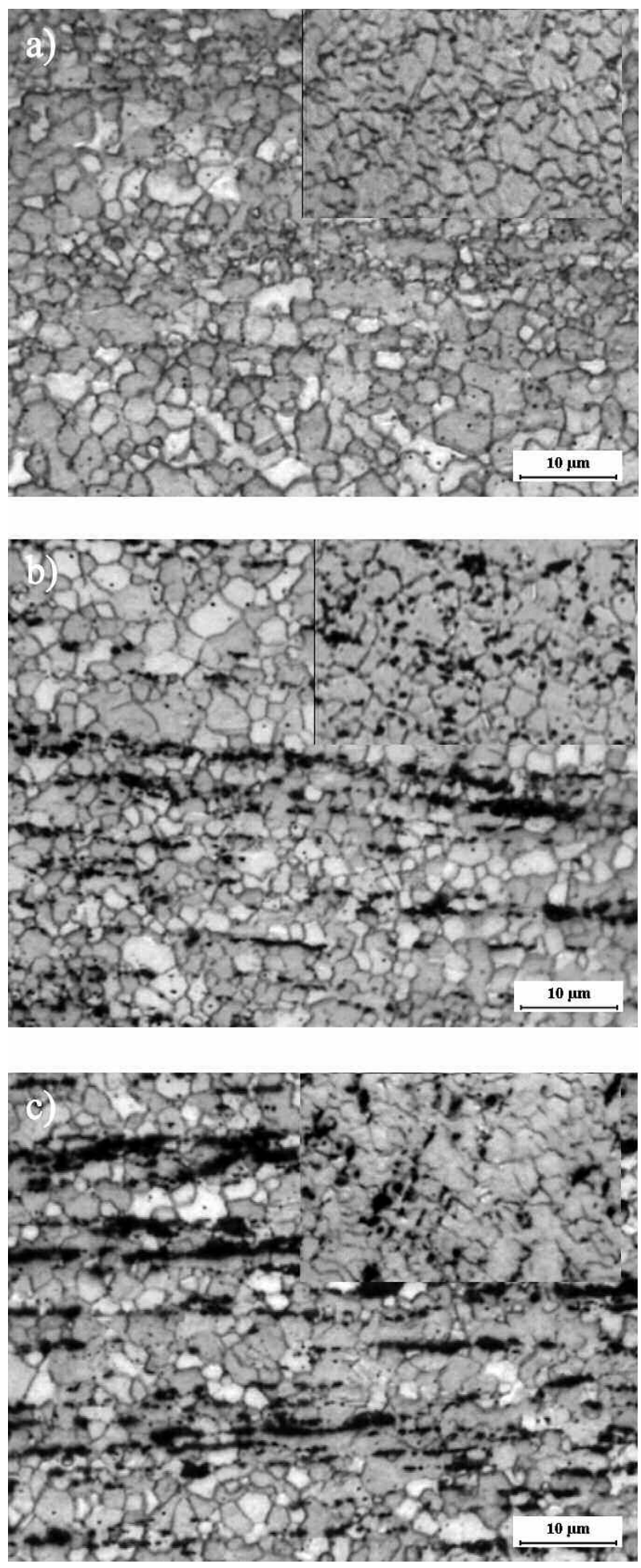

Figura 2. Micrografía óptica mostrando las secciones longitudinales de las barras extruidas. EI recuadro del interior es un detalle, a los mismos aumentos, de las secciones transversales: a) AZ31. b) AZ31-1 \% C. c) AZ31-2 \% C.

Figure 2. Optical micrograph showing longitudinal section of extruded bars. The inset is a detail of transversal section at the same magnification. a) AZ31. b) AZ31-1 \% C. c) AZ31-2 \% C. 


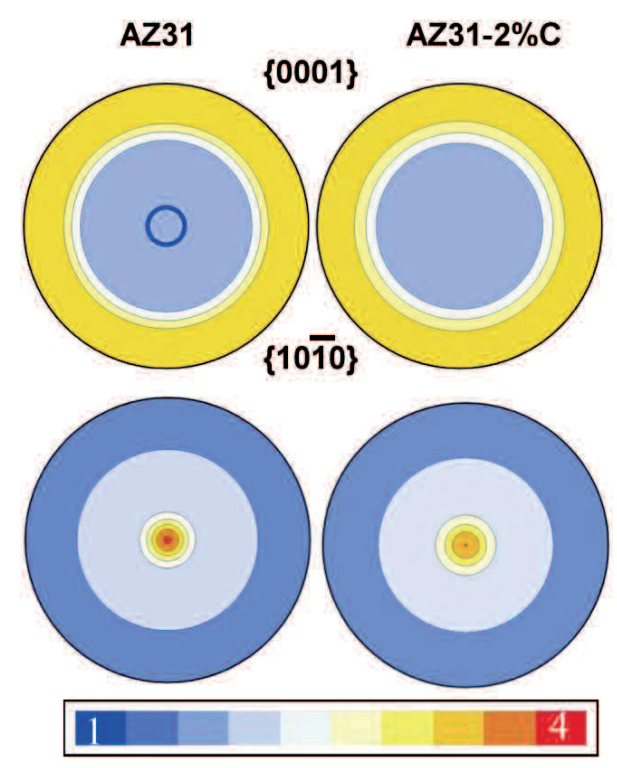

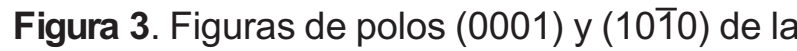
aleación AZ31 y AZ31-2 \% C.

Figure 3. (0001) and (1010) pole figures of AZ31 and $A Z 31-2 \% C$.

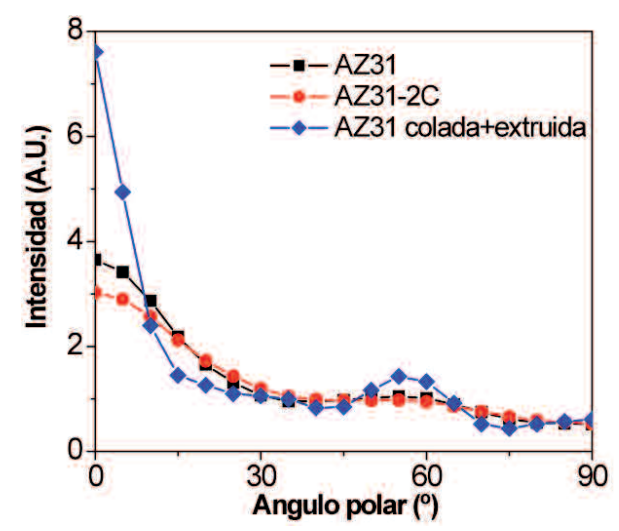

Figura 4. Intensidad de la figura de polos de los planos (1010) en función del ángulo polar.

Figure 4. Pole figure intensity of the (1010) planes as function of the polar angle $(\Theta)$.

en la tabla I. A temperaturas bajas, Tambiente y $50^{\circ} \mathrm{C}$, los tres materiales presentan elevados valores de límite elástico, debido al pequeño tamaño de grano. El hecho de que la aleación sin reforzar alcance valores ligeramente superiores ha de asociarse a pequeños efectos de la textura. A temperaturas intermedias $\left(100^{\circ} \mathrm{C}\right)$, sin embargo, las nanofibras parecen ejercer un efecto de refuerzo, alcanzando los materiales reforzados valores de límite elástico y resistencia un $30 \%$ superiores a los de la aleación sin

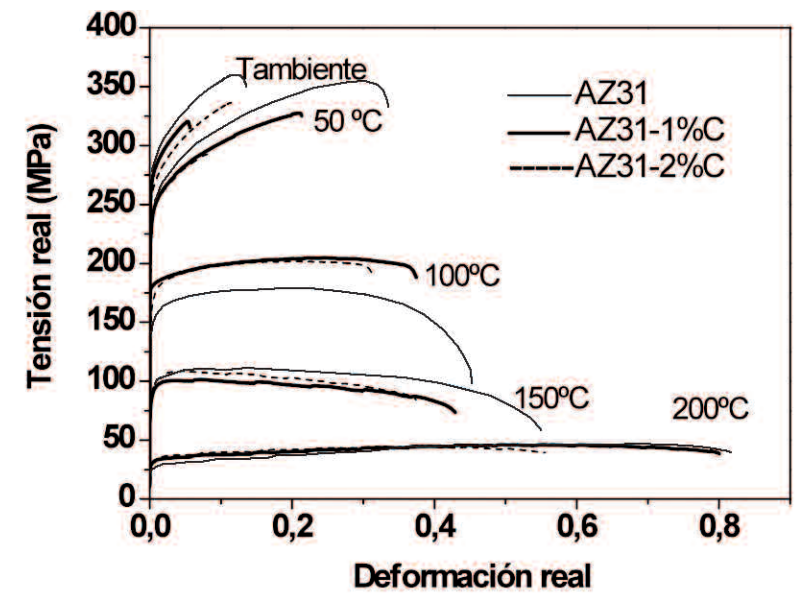

Figura 5. Curvas tensión real-deformación real de los tres materiales ensayados a diferentes temperaturas.

Figure 5. True stress-true strain curves of the three materials at different tests temperatures.

Tabla I. Valores medios de limite elástico, LE (MPa), resistencia a la tracción, Rm (MPa) y alargamiento a rotura, $\varepsilon(\%)$, de los tres materiales ensayados a tracción, a diferentes temperaturas

Table I. Mean values of the yield stress, LE (MPa), tensile strength, Rm (MPa), and elongation to failure, $\varepsilon(\%)$, of the three materials tested at different temperatures

\begin{tabular}{ccrrr}
\hline Material & Temp. & LE & Rm & $\varepsilon$ \\
\hline & Ambiente & 272 & 360 & 12,5 \\
& $50{ }^{\circ} \mathrm{C}$ & 230 & 355 & 29 \\
AZ31 & $100^{\circ} \mathrm{C}$ & 134 & 179 & 45 \\
& $150{ }^{\circ} \mathrm{C}$ & 87 & 110 & 51 \\
& $200{ }^{\circ} \mathrm{C}$ & 23 & 46 & 80 \\
& & & & \\
& Ambiente & 265 & 319 & 5,5 \\
AZ31-1\% C & $50{ }^{\circ} \mathrm{C}$ & 229 & 327 & 21 \\
& $100{ }^{\circ} \mathrm{C}$ & 179 & 204 & 37 \\
& $150{ }^{\circ} \mathrm{C}$ & 83 & 103 & 42 \\
& $200{ }^{\circ} \mathrm{C}$ & 29 & 45 & 80 \\
& & & & \\
& Ambiente & 255 & 337 & 11,5 \\
& $50{ }^{\circ} \mathrm{C}$ & 232 & 292 & 8 \\
AZ31-2 \% C & $100{ }^{\circ} \mathrm{C}$ & 156 & 201 & 31 \\
& $150{ }^{\circ} \mathrm{C}$ & 85 & 108 & 36 \\
& $200^{\circ} \mathrm{C}$ & 28 & 44 & 80 \\
\hline
\end{tabular}


reforzar, pero con una ductilidad apreciable, aunque menor que la del material sin reforzar. A partir de $150{ }^{\circ} \mathrm{C}$ los tres materiales muestran, de nuevo, un mismo comportamiento caracterizado por una gran disminución de su resistencia que se asocia a la activación de los sistemas de deslizamiento piramidal y prismático y a la recristalización dinámica.

Se han estudiado las superficies de fractura de los materiales ensayados a temperatura ambiente, $50 \mathrm{y}$ $100^{\circ} \mathrm{C}$. A bajos aumentos, todos los materiales presentan una fractura similar, con mayor deformación plástica a medida que la temperatura de ensayo es mayor. Así, a $100^{\circ} \mathrm{C}$, la fractura es intergranular, pero la de los materiales reforzados parece algo más frágil, como se puede observar en las áreas señaladas con flechas, de la figura 6. La naturaleza intergranular de la fractura se pone más claramente de manifiesto en la imagen de la figura 7, correspondiente a la superficie de una probeta AZ31-1 \% C, ensayada a $100^{\circ} \mathrm{C}$, donde se observa que la fractura ocurre de modo intergranular por coalescencia de microcavidades. También, se observan grietas transgranulares.

Hay diferentes autores que indican que una mala dispersión de las nanofibras de carbono en una matriz de magnesio causa una disminución de su resistencia por la aparición de poros y defectos en el material ${ }^{[1]}$. Otra posible causa del fallo de estos materiales se atribuye, también, a la formación de carburo de aluminio y magnesio en la intercara matriz/nanofibra. Esto se ha observado cuando la temperatura es superior a $500{ }^{\circ} \mathrm{C}$ y el contenido de aluminio en la aleación de magnesio supera el 0,6 \%. Ninguno de estos problemas parece ser el caso de los materiales compuestos procesados en este trabajo ya que en el caso de los ensayos de tracción a temperatura ambiente y $50^{\circ} \mathrm{C}$, no se produce una disminución importante de los valores de límite elástico y resistencia mecánica. Además, el estudio de las superficies de fractura no ha mostrado fallo por la intercara matriz/nanofibras.

Otra posible causa que explicaría la mayor resistencia de la aleación monolítica a temperaturas inferiores a $100^{\circ} \mathrm{C}$ sería el efecto de la textura, aspecto muy importante a considerar en el reforzamiento de las aleaciones de magnesio. El hecho de que, a temperaturas inferiores a $100^{\circ} \mathrm{C}$, los materiales compuestos presenten valores de limite elástico y resistencia algo menores a los de la aleación sin reforzar puede estar relacionado con las ligeras diferencias en textura encontradas. Esto es razonable ya que las nanofibras pueden actuar obstaculizando la rotación de los granos durante el proceso de extrusión. Aunque, como se ha comentado anteriormente, todos los materiales presentan una textura de fibra débil, se ha comprobado, experimentalmente (Figs. 3 y 4), que es ligeramente superior la del material monolítico.
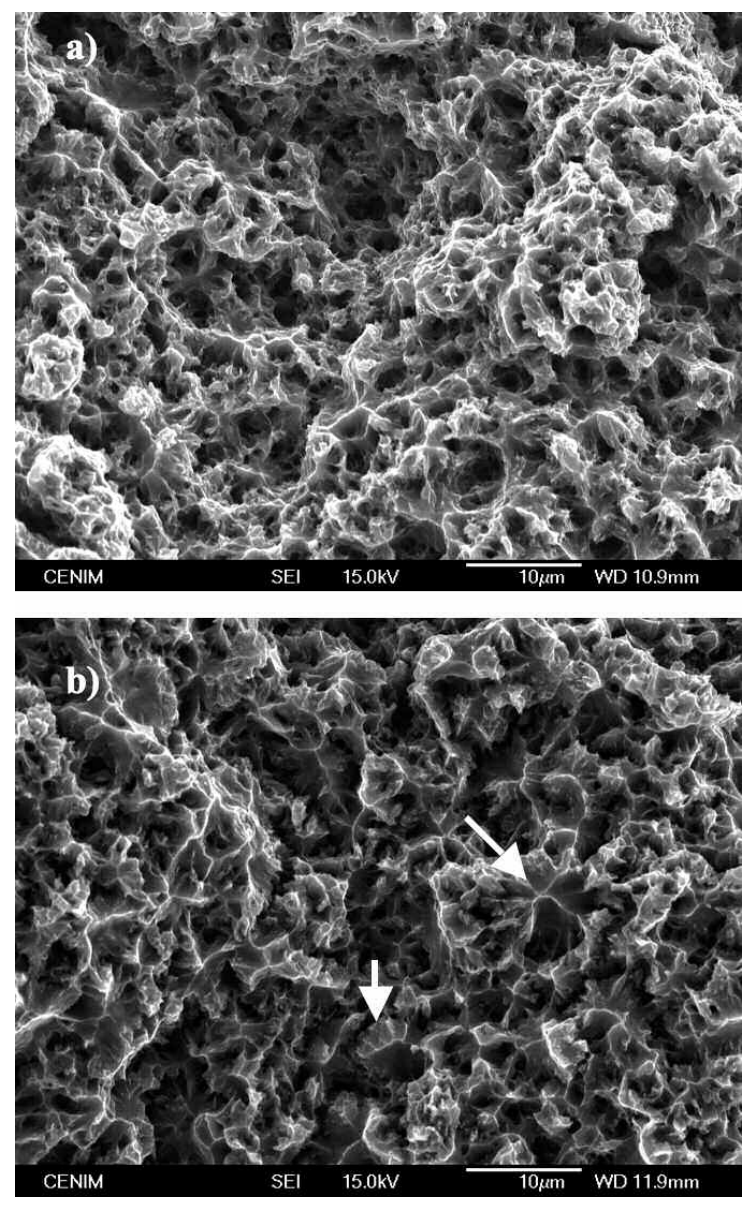

Figura 6. Superficies de fractura de los materiales ensayados a $100{ }^{\circ} \mathrm{C}$. a) AZ31. b) AZ31$1 \% \mathrm{C}$.

Figure 6. Fracture surfaces of the materials tensile tested at $100^{\circ} \mathrm{C}$. a) AZ31. b) AZ31-1 \% C.

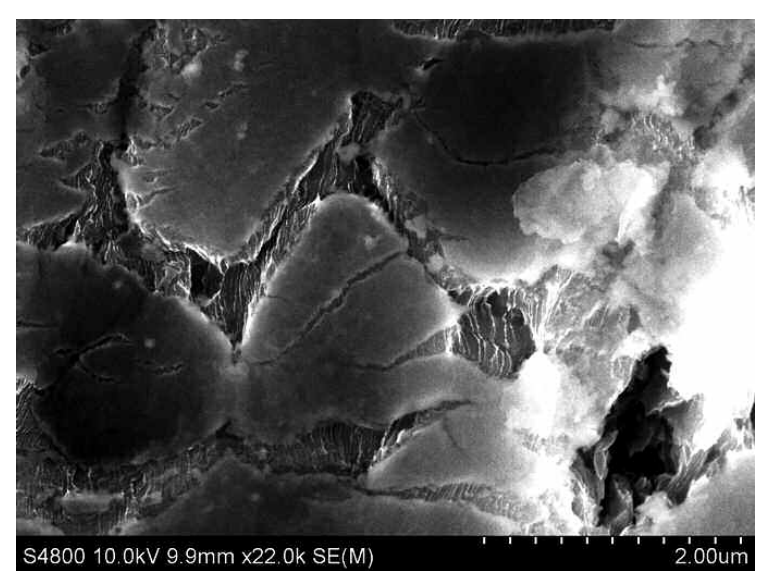

Figura 7. Superficie lateral de una probeta AZ31$1 \%$ C ensayada a $100{ }^{\circ} \mathrm{C}$. Zona próxima a la superficie de fractura.

Figure 7. External surface of AZ31-1\% C sample tested at $100^{\circ} \mathrm{C}$. Zone next to the fracture 
Aunque, de las micrografías presentadas en la figura 2, resulta evidente que las nanofibras están distribuidas como aglomerados de diferente tamaño, que en algunos casos alcanzan algunos micrómetros y están situadas preferentemente en las fronteras originales de las partículas de polvo de magnesio, se ha obtenido una mejora de las propiedades mecánicas, a $100^{\circ} \mathrm{C}$. Ello parece indicar que la introducción de nanofibras de carbono refuerza mecánicamente la aleación y, concretamente, las fronteras de grano, a esta temperatura. La razón de esta aseveración se basa en los siguientes hechos: todos los materiales tienen el mismo tamaño de grano, independientemente de la presencia o no de nanofibras y de la fracción en volumen de éstas. Además, la menor ductilidad que muestran los materiales reforzados es una indicación de la disminución de la movilidad de dislocaciones. No obstante, sí se ha puesto de manifiesto que el refuerzo, con el 2 \% de carbono, no produce un aumento de los valores de límite elástico y resistencia mecánica sino, más bien una disminución. Estos resultados están de acuerdo con lo observado por otros autores, los cuales indican que, cuando se añade más del 1,5 \% de nanotubos, incluso utilizando procesos de fabricación más complejos que los utilizados en este estudio, se produce un deterioro del material y lo atribuyen a la formación de agregados de nanotubos que generan $\operatorname{poros}^{[1]}$.

Tal y como se ha comentado en la introducción, este trabajo es un estudio preliminar y, como tal, quedan aún muchas cuestiones que explicar. La más importante es conocer por qué las nanofibras refuerzan a $100^{\circ} \mathrm{C}$. Para ello, será necesario realizar un estudio mediante TEM de las muestras ensayadas para analizar la interacción nanofibras/dislocaciones.

\section{CONCLUSIONES}

Del estudio llevado a cabo sobre la influencia que tiene, en las propiedades mecánicas de la aleación AZ31 procesada por una ruta pulvimetalúrgica convencional, la adición del 1 \% y 2 \% (fracción en volumen) de nanofibras de carbono, se puede concluir lo siguiente:

- La presencia de las nanofibras no afecta al tamaño de grano de los materiales extruidos. Los tres materiales, AZ31, AZ31-1 \% C y AZ31$2 \% \mathrm{C}$, muestran una estructura completamente recristalizada con un tamaño de grano inferior a $5 \mathrm{~mm}$, algo heterogéneo.

- El refuerzo, en forma de aglomerados de diferentes tamaños, se alinea en la dirección de extrusión y está distribuido, preferentemente, en las fronteras originales de las partículas de polvo.

- Los tres materiales presentan una textura de fibra débil, con el plano (0001) paralelo a la dirección de extrusión. El refuerzo debilita, ligeramente, la textura de extrusión.

- Respecto a las propiedades mecánicas, la aleación sin reforzar es la que presenta valores más altos de límite elástico, resistencia mecánica y alargamiento a Tambiente y $50{ }^{\circ} \mathrm{C}$. A temperaturas intermedias, sin embargo, las nanofibras ejercen un efecto de refuerzo, alcanzando los materiales compuestos valores de límite elástico y resistencia un 30 \% superiores a los de la aleación sin reforzar y con una ductilidad apreciable.

- La fracción en volumen de nanofibras, superior al $1 \%$, produce una ligera disminución de la resistencia mecánica del material.

\section{Agradecimientos}

Este trabajo está financiado por el Ministerio de Ciencia e Innovación a través del Proyecto MAT 2009-07811. Los autores agradecen al Grupo AntolinIrausa, S.A, el suministro de las nanofibras.

\section{REFERENCIAS}

[1] Y. Shimizu, S. Miki, T. Soga, I. Itoh, H. Todoroki, T. Hosono, K. Sakaki, T. Hayashi, Y.A. Kim, M. Endo, S. Morimotob y A. Koide. Scripta Mater. 58 (2008) 267-270.

[2] Q. Li, A. Viereckl, C.A. Rottmair y R.F. Singer, Compos. Sci. Technol. 69 (2009) 1.193-1.199.

[3] T. Honma, K. Nagai, A. Katou, K. Arai, M. Suganum y S. Kamadoa, Scripta Mater. 60 (2009) 451-454.

[4] R. George K.T. Kasthyap, R. Rhaul y S. Yamdagni. Scripta Mater. 58 (2005) 1.159-1.163.

[5] S. Kleiner y P.J. Uggowitzer, Mater. Sci.Eng. A 379 (2004) 258-263. 